\title{
Checkpoint Inhibitors in Squamous Non-Small Cell Lung Cancer (NSCLC)
}

\author{
Bakulesh Khamar* \\ R\&D Department, Cadila Pharmaceuticals Limited, Ahmedabad, India
}

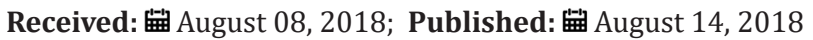

*Corresponding author: Bakulesh Khamar, Cadila Pharmaceuticals Limited, Cadila Corporate Campus Sarkhej-Dholka Road, Bhat, Ahmedabad, Gujarat, India

\begin{abstract}
Checkpoint inhibitors have demonstrated efficacy in squamous NSCLC with tolerable toxicity profile. They are used as a monotherapy, in combination with chemotherapy or following chemo-radiotherapy as a first line, second line or consolidation therapy. As a first line, Pembrolizumab improves outcome (PFS) as a monotherapy in patients with PD-L1 expression of $\geq 50 \%$ (HR; 0.35). When used with chemotherapy, it improves PFS (HR; 0.56) irrespective of PD-L1 expression level. Nivolumab also improves PFS (HR; 0.62), when used as a second line for those progressing with chemotherapy. Durvalumab improves PFS (HR; 0.68) when used following chemo-radio therapy as a consolidation therapy.
\end{abstract}

Keywords: Checkpoint Inhibitors; Pembrolizumab; Nivolumab; Atezolizumab; Durvalumab; PD-L1; Squamous NSCLC; Progression Free Survival; Overall Survival

\section{Introduction}

Lung cancer remains the most common cancer diagnosis and the leading cause of cancer-related deaths, with approximately 1.8 million new cases and 1.59 million deaths accounting every year worldwide [1]. Non-small cell lung cancer (NSCLC) covers the $80 \%$ of these lung cancer cases and mainly includes two large histological subsets, adenocarcinoma and squamous non-small cell lung cancer (Sq NSCLC) Clinically also Sq NSCLC differs remarkably from adenocarcinoma as it is located more centrally, and arise segmentally involving lobar or main bronchi and often having central cavitation. There is difference in molecular markers also. Based on molecular changes targeted therapies have been developed for subtypes of adenocarcinoma of lung e.g. EGFR inhibitors. Their use is associated with improved outcome with better tolerability. However, such advances have not taken place in management of Sq NSCLC and the conventional platinum-based chemotherapy remained the first line and second line option for patients with Sq NSCLC till recently. Necitumab approved for Sq NSCLC did not became popular due to its side effect profile and limited benefit [2]. Introduction and development of checkpoint inhibitors has changed the management of Sq NSCLC. Checkpoint inhibitors have been evaluated in NSCLC in whole spectrum ranging from neoadjuvant to third line settings and has been indicated as first line, second line and consolidation therapy [2,3]. In this review, information related to use of check point inhibitor in the treatment of Sq NSCLC is presented. Pembrolizumab (Keynote-407), Atezolizumab (IMpower-131) have been evaluated with chemotherapy in first line management of advanced Sq NSCLC. Nivolumab (CheckMate 017) has been evaluated in second line management of Sq NSCLC as monotherapy. Rest of studies evaluated checkpoint inhibitors in management of Sq NSCLC as a subgroup in main study. They are also included in this review.

\section{Checkpoint Inhibitors as a Neoadjuvant Therapy}

Nivolumab has been evaluated in neoadjuvant setting prior surgical resection in 21 patients [4]. All patients received two doses of $3 \mathrm{mg} / \mathrm{kg}$ of nivolumab, two weeks apart. Of 6 patients included in the study, two had pathological response. This was associated with lymphocytic infiltration of tumor. There was higher frequency of T-cell clones that were shared between intratumoral and peripheral compartments and a higher clonality of the T-cell population. Many of these clones were not detected in the peripheral blood before treatment. Tumor response to nivolumab was also related to tumor mutation burden. 


\section{Checkpoint Inhibitors as a First Line Therapy}

Checkpoint inhibitors have been evaluated as a monotherapy in patients having PD-L1 expression Tumor Proportion Score (TPS) $\geq 50 \%$ as well as in patients with high mutation burden. In all, the majority of the Task Force recommended pembrolizumab monotherapy for patients with Sq NSCLC and PD-L1 TPS $\geq 50 \%$, based on Level A evidence. For patients with squamous histology and PD-L1 TPS $<50 \%$, the Task Force unanimously recommended combination pembrolizumab + chemotherapy pending FDA approval, based on Level A evidence.

\section{Monotherapy}

\section{Pembrolizumab}

Pembrolizumab as monotherapy has been compared against first line chemotherapy in KEYNOTE-024 and KEYNOTE-042 clinical studies. KEYNOTE -024 enrolled patients with PD-L1 expression level $\geq 50 \%$ while KEYNOTE-042 enrolled patients with PD-L1 expression level $\geq 01 \%$.

\section{Keynote-024 [5]}

305 patients, were randomized to receive pembrolizumab (200 mg fixed dose every 3 weeks) or investigator's choice of platinum-based chemotherapy. Patients eligible for EGFR inhibitors or ALK inhibitors were excluded. It included 56 with Sq NSCLC histology 18\% in each arm) expressing more than 50\% PD-L1. Progression Free Survival (PFS) was significantly better in the Sq NSCLC subpopulation receiving immunotherapy compared to chemotherapy [Hazard Ratio (HR) $=0.35,95 \%$ Confidence Interval (CI): 0.17-0.71]. This was also better than the improvement seen with pembrolizumab in whole group [HR 0.5 for group vs 0.35 for Sq NSCLC]. Similar to other immunotherapies, pembrolizumab appeared fewer toxicities compared to chemotherapy arm (any grade $73.4 \%$ vs. $90.0 \%$ and grade $3 / 426.6 \%$ vs. $53.3 \%$, respectively). Most immunotherapy-related adverse events (AEs) were early detected at grade 1 or 2 and well managed with no recorded immunotherapy-related deaths.

\section{Keynote-042 [6]}

1274 patients, were randomized to receive pembrolizumab (200 mg fixed dose every 3 weeks) or investigator's choice of platinum-based chemotherapy. Patients eligible for EGFR inhibitors or ALK inhibitors were excluded. It included 492 patients with Sq NSCLC histology of which 243 received pembrolizumab. Overall Survival (OS) was significantly better in the Sq NSCLC subpopulation receiving immunotherapy compared to chemotherapy [HR $=0.75$, 95\% CI: 0.60-0.93]. This was also better than the improvement seen with pembrolizumab in non-squamous NSCLC [HR 0.86; 95\% CI: 0.72-1.03]

\section{Nivolumab}

Nivolumab has been evaluated as first line therapy in checkmate 227 and checkmate 026 .

\section{Checkmate227 [7]}

Combination of checkpoint inhibitors (Nivolumab + Iplimumab) was evaluated against chemotherapy in patients with advanced NSCLC (squamous and non-squamous) in the CheckMate227 study in first line setting. All patients had high tumor mutational burden [more than 10]. It had 100 patients with Sq NSCLC and 199 patients with non-squamous NSCLC. HR for disease progression or death for squamous NSCLC was 0.63 (95\% CI, 0.39-1.04). It was lower $(0.63$ vs 0.55 ) compared to non-squamous NSCLC as well as compared to whole group [ 0.63 vs 0.58 ]. Survival data is immature.

\section{Checkmate026 [8]}

CheckMate026 evaluated Nivolumab as first line therapy compared chemotherapy in 541 untreated patients having advanced NSCLC with PD-L1 expression >1\% .It included 130 patients with Sq NSCLC. Trial failed to demonstrate significant advantage of nivolumab in whole group analysis or any subgroup analysis. Though not significant, Nivolumab improved median PFS by 0.5 months [HR; 0.83 (95\% CI, 0.54-1.26)] and median OS by 0.3 months [HR; 0.82 (95\% CI0.54-1.24)] in patients with Sq NSCLC. In non-squamous NSCLS, chemotherapy performed better (Table 1).

Table 1: First line therapy: Checkpoint inhibitors as monotherapy.

\begin{tabular}{|c|c|c|c|c|}
\hline Study* & Comparison & Selection & PFS HR $(95 \% \mathrm{CI})$ & OS HR $(95 \% \mathrm{CI})$ \\
\hline $\begin{array}{l}\text { KEYNOTE-024* } \\
(n=56)\end{array}$ & $\begin{array}{l}\text { Pembrolizumab vs. } \\
\text { platinum doublet }\end{array}$ & PD-L1 $\geq 50 \%$ & $\begin{array}{l}\mathrm{HR}=0.35, \\
(0.17-0.71)\end{array}$ & \\
\hline $\begin{array}{l}\text { KEYNOTE- 042* } \\
\quad(n=243)\end{array}$ & $\begin{array}{l}\text { Pembrolizumab vs. } \\
\text { platinum doublet }\end{array}$ & PD-L1 $\geq 1 \%$ & & $\mathrm{HR}=0.75,(0.60-0.97)$ \\
\hline $\begin{array}{l}\text { CheckMate } 227^{*} \\
\qquad(\mathrm{n}=100)\end{array}$ & $\begin{array}{l}\text { Nivolumab + Yervoy vs. } \\
\text { platinum doublet }\end{array}$ & $\begin{array}{c}\text { TMB-high } \geq 10 \text { mutations/ } \\
\text { megabase }\end{array}$ & $\begin{array}{c}\mathrm{HR}=0.63, \\
(0.39-1.04)\end{array}$ & Immature \\
\hline $\begin{array}{l}\text { CheckMate 026* } \\
\qquad(n=130)\end{array}$ & $\begin{array}{c}\text { Nivolumab vs platinum } \\
\text { doublet }\end{array}$ & PD-L1 $\geq 1 \%$ & $\begin{array}{c}\mathrm{HR}=0.83, \\
(0.54-1.26)\end{array}$ & $\begin{array}{l}\mathrm{HR}=0.81, \\
(0.54-1.24)\end{array}$ \\
\hline
\end{tabular}

\section{Combination with Chemotherapy}

Checkpoint inhibitors have been evaluated with chemotherapy in management of squamous as well as non-squamous NSCLC.
For Sq NSCLC, Pembrolizumab as well as atezolizumab has been evaluated in combination with paclitaxel containing platinum doublet. Both are associated with improved outcome. 


\section{Pembrolizumab [9]}

Pembrolizumab in combination with carboplatin + nabpaclitaxel/paclitaxel as first-line treatment was evaluated in KEYNOTE-407 clinical trial in 559 treatment naïve patients with advanced, Sq NSCLC. Combination was associated with increase in response rate by $23.4 \%$ [Objective Response Rate (ORR) $58.4 \%$ vs. $35 \%, \mathrm{p}<0.01]$. The duration of response was also more durable [7.7 versus 4.8 months ].Improved response rate was also associated with improved PFS by 1.6 months [ 6.4 months vs 4.8 months; $\mathrm{HR}=0.56$; $95 \% \mathrm{CI}, 0.45-0.70 ; \mathrm{p}<0.01]$ and improved $\mathrm{OS}$ by 4.6 months (15.9 months vs 11.3 months; $\mathrm{HR}=0.64$, 95\% CI: 0.49 $0.85, \mathrm{p}=0.0008$ ) in patients who were treated with combination pembrolizumab + chemotherapy compared to patients treated with chemotherapy alone Additional, OS benefit was seen irrespective of tumor PD-L1 status (TPS < 1\%, HR = 0.61 [95\% CI: 0.35-0.98]; TPS $1-49 \%$, HR $=0.57$ [95\% CI: $0.36-0.90]$; TPS $>50 \%, \mathrm{HR}=0.64$ [95\% CI: 0.37-1.10]). Grade 3-5 AEs were comparable across the pembrolizumab/chemotherapy and placebo cohorts $169.8 \%$ vs. $68.2 \%$, respectively). PD-L1 status was measured by tumor proportion score (TPS) was identical in both arms. It was $34.2 \%$ vs. $35.2 \%$ for TPS $<1 \%, 37.1 \%$ vs. $35.2 \%$, for TPS $1 \%-49 \%$, and $26.1 \%$ vs. $26 \%$ TPS $\geq 50 \%$. The HR for OS was $0.61,0.57$, and 0.64 favouring the pembrolizumab arm in the TPS $<1 \%,<1 \%-49 \%$, and Table 2: First line therapy: Checkpoint inhibitors with chemotherapy.

\begin{tabular}{|c|c|c|c|c|}
\hline Study & Comparision & ORR & $\begin{array}{c}\text { PFS } \\
\text { months } \\
\text { HR (95\% CI) } \\
\text { P }\end{array}$ & $\begin{array}{c}\text { OS } \\
\text { months } \\
\text { HR }(95 \% \mathrm{CI}) \\
\text { P }\end{array}$ \\
\hline $\begin{array}{l}\text { KEYNOTE-407 } \\
(n=559)\end{array}$ & $\begin{array}{c}\text { Carboplatin (nab-paclitaxel/ } \\
\text { paclitaxel) +/- } \\
\text { Pembrolizumab }\end{array}$ & $\begin{array}{c}58 \% \text { vs. } 35 \% \\
\text { p }<0.01\end{array}$ & $\begin{array}{c}1.6 \text { months } \\
\text { HR=0.56; } \\
(0.45-0.70) \\
\text { p<0.01 }\end{array}$ & $\begin{array}{c}4.6 \text { months } \\
\text { HR }=0.64 \\
(0.49-0.85) \\
p=0.0008\end{array}$ \\
\hline $\begin{array}{l}\text { IMpower131 } \\
\qquad(n=686)\end{array}$ & $\begin{array}{c}\text { Carboplatin (nab- } \\
\text { paclitaxel/ paclitaxel) +/- } \\
\text { Atezolizumab }\end{array}$ & $49 \%$ vs. $41 \%$ & $\begin{array}{l}0.7 \text { months } \\
\text { HR = 0.71; } \\
(0.60-0.85) \\
p<0.0001\end{array}$ & $\begin{array}{c}9.5 \text { months } \\
\text { Only in } \\
\text { with high-PD-L1 } \\
\text { HR }=0.56 \\
(0.32-0.99) \\
\text { No improvement } \\
\text { rest }\end{array}$ \\
\hline $\begin{array}{l}\text { CheckMate } 227^{*} \\
\qquad(n=49)\end{array}$ & $\begin{array}{l}\text { Platinum doublet } \pm \\
\text { Nivolumab }\end{array}$ & - & $\mathrm{HR}=0.92$ & Immature \\
\hline
\end{tabular}

\section{Checkpoint inhibitors as a Consolidation therapy}

Checkpoint inhibitors has been evaluated as a consolidation therapy following chemoradiotherapy.

\section{Durvalumab [12]}

In a PACIFIC study, 713 patients with stage III NSCLC nonprogressing after at least two cycles of chemo-radiation were randomized to receive durvalumab $(n=476)$ at $10 \mathrm{mg} / \mathrm{kg}$ or placebo (273), every other week, for up to a year as consolidation/ $\geq 50 \%$ subgroups, respectively. Across the same 3 subgroups, the HR for PFS favouring the pembrolizumab arm was $0.68,0.56$, and 0.37 , respectively.

\section{Atezolizumab [10]}

IMpower131 study is evaluating atezolizumab + carboplatin \& nab-paclitaxel in patients with advanced Sq NSCLC. Addition of atezolizumab improved response rate by $8 \%$ ( $49 \%$ vs $41 \%$ ). Improvement in PFS was 0.7 months (6.3 months vs 5.6 months, $\mathrm{HR}=0.71$ [95\% CI: 0.60-0.85], $\mathrm{p}<0.0001$ ). Improvement in OS was of 9.5 months in patients with high-PD-L1, (23.6 months vs 14.1 months, HR $=0.56$ [95\% CI: 0.32-0.99]. No improvement in OS was seen remaining patients with low [12.4 months vs 16.6 months, HR $=1.34$ [95\% CI: 0.95-1.90] or no PD-L1 expression (13.8 months vs 12.5 months, $\mathrm{HR}=0.86$ [95\% CI: 0.65-1.15]).

\section{Nivolumab [11]}

CheckMate227 also evaluated nivolumab + chemotherapy against chemotherapy as well as against nivolumab + iplimumab in 550 patients. It included 49 patients randomised to receive nivolumab + chemotherapy or chemotherapy. PFS with Nivolumab + CT was found to be little better (HR=0.92) which was not significant. Patients with non-squamous NSCLC did better (HR=0.68) (Table 2). maintenance therapy following chemo-radiation. It included 326 patients with Squamous NSCLC. 224 patients with squamous NSCLC received durvalumab and 102 received placebo. Durvalumab was associated improved progression free survival. The hazard ratio was 0.45 (0.33-0.59) for adenocarcinoma and $0.68(0.50-0.92)$ for squamous NSCLC. Thus Durvalumab provides better outcome for adenocarcinoma compared to Squamous NSCLC. The safety profile of durvalumab was similar to placebo regarding toxicities greater than grade 3 (29.9\% vs. $26.1 \%$, and the most commonly observed pneumonia presented in $4.4 \%$ vs. $3.8 \%$ of cases, respectively). 


\section{Pembrolizumab [13]}

Pembrolizumab was evaluated in 92 patients without progression following chemoradiotherapy in Lun14-179 (a single arm) study. 55 (59.8\%) had stage IIIA and 37 (40.2\%) had IIIB disease. It included 40 (43.5\%) patients with Sq NSCLC. All received pembrolizumab $200 \mathrm{mg}$ IV q3wk for up to 1 year. Separate subgroup analysis for Sq. NSCLC is not available. For 92 patients, median time to distant metastasis or death was 22.4 months (95\% CI; 17.9- NR). Median PFS was 17.0 months (95\% CI; 11.9-NR)

\section{Checkpoint Inhibitors as a Second Line Therapy}

Checkpoint inhibitors (nivolumab, pembrolizumab and atezolizumab) had their first approval in management of NSCLC as a second line therapy based on improvement in OS. They were evaluated as a monotherapy against docetaxel as a chemotherapy. Improvement in OS was not related to changes in PFS. Surprisingly, all three checkpoint inhibitors were found to provide less benefit in squamous NSCLC compared to advantage seen in non-squamous NSCLC. Atezolizumab was found inferior to Docetaxel in Sq NSCLC subgroup.

\section{Nivolumab}

Nivolumab was the first immunotherapy agent that received approval for the second line approach of Sq NSCLC, based on the results of CheckMate017. This phase III, randomized trial exclusively enrolled 272 patients with Sq NSCLC and compared nivolumab versus docetaxel in the second line setting. All enrolled patient had received a platinum doublet as a first line [14]. This phase III, randomized trial resulted in a statistically significant improvement of ORR (20\% versus 9\%). Nivolumab improved median OS by 3.2 months (9.2 vs. 6.0 months; HR 0.59; 95\% CI: 0.44-0.79; p<0.001) [14]. This was also associated with increased survival of $18 \%$ [42\% vs $24 \%$ ] at one year and $16 \%$ [24\% vs $8 \%$ ] at end of two years. PDL1 expression was not associated with efficacy of nivolumab and notably, no survival or response advantage was found in different levels of PD-L1 (1\%, 5\% and 10\%) [14]. Nivolumab was found to have a better safety profile than docetaxel. Grade 3-4 toxicities were seen $7 \%$ of patients receiving nivolumab compared to $55 \%$ receiving Docetaxel. All grade toxicities were also lower with nivolumab (59\% vs. 87\%) [14]. Two-year overall survival rates with nivolumab versus docetaxel were $23 \%$ versus $8 \%$ in Sq NSCLC [15]. For sake of convenience and reduce hospital visits, Nivolumab $480 \mathrm{mg}$ IV can be given at every 4 weeks. It is demonstrated to be equally effective [16].

\section{Nivolumab in Elderly}

Nivolumab has also been evaluated as a monotherapy in a single arm study in 371 elderly patients with Sq NSCLC following progression [17]. Of these 126 (34\%), 175(47\%) and 70 (19\%) were aged $<65,65-<75$ and $\geq 75$ years, respectively. The response rate is identical [18\%-19\%] across all age groups. Median PFS was identical up to age of 75 . However, it was lower for patients above 75 years of age [17]. Median OS was 8.6 months for patients aged $<65$ years, 8.0 months for patients aged $65-<75$ years and 5.8 months for patients aged $65-<75$ years [17].

\section{Pembrolizumab}

Keynote-010 evaluated pembrolizumab in 1034 previously treated patients (second line) with a PD-L1 TPS $\geq 1 \%$. It compared pembrolizumab (2 mg/kg or $10 \mathrm{mg} / \mathrm{kg}$ ) with docetaxel [18]. Of 1034 recruited patients 222 had squamous NSCLC [18]. There was improvement in overall survival in patients receiving pemrolizumab compared to docetaxel in patients with Sq NSCLC [HR= 0.74; 95\% CI $0.50-1.09$ ] [18]. This was lower than survival benefit [HR= 0.63; 95\% CI $0.50-0.079$ ] seen in non-squamous NSCLC [18].

\section{Atezolizumab}

Table 3: Second line therapy: Checkpoint inhibitors as monotherapy.

\begin{tabular}{|c|c|c|c|c|c|c|c|}
\hline \multirow[b]{2}{*}{ Study } & \multirow[b]{2}{*}{ Comparison } & \multicolumn{3}{|c|}{ Overall survival } & \multicolumn{3}{|c|}{ Progression-free survival } \\
\hline & & Months & $\begin{array}{c}\text { HR } \\
(95 \% \mathrm{Cl})\end{array}$ & $P$ value & Months & $\begin{array}{c}\text { HR } \\
(95 \% \mathrm{Cl})\end{array}$ & $P$ value \\
\hline $\begin{array}{l}\text { CheckMate } 017 \\
\qquad(\mathrm{n}=272)\end{array}$ & $\begin{array}{c}\text { Nivolumab } \\
\text { vs. Docetaxel }\end{array}$ & 3.2 & $\begin{array}{c}0.59 \\
(0.44-0.79)\end{array}$ & $\mathrm{p}<0.001$ & 0.7 & $\begin{array}{c}0.62 \\
(0.47-0.81)\end{array}$ & $p<0.001$ \\
\hline $\begin{array}{l}\text { KEYNOTE-010* } \\
(\mathrm{n}=222)\end{array}$ & $\begin{array}{l}\text { Pembrolizumab } \\
\text { vs. Docetaxel }\end{array}$ & 1.2 & $\begin{array}{c}0.74 \\
(0.50-1.09)\end{array}$ & -- & - & - & - \\
\hline $\begin{array}{l}\text { Poplar* } \\
(\mathrm{n}=97)\end{array}$ & $\begin{array}{l}\text { Atezolizumab } \\
\text { vs. Docetaxel }\end{array}$ & 1.5 & $\begin{array}{c}0.8 \\
(0.49-1.30)\end{array}$ & - & - & - & - \\
\hline $\begin{array}{c}\text { OAK* }^{*} \\
(n=222)\end{array}$ & $\begin{array}{l}\text { Atezolizumab } \\
\text { vs. Docetaxel }\end{array}$ & -1.2 & $\begin{array}{c}0.73 \\
(0.54-0.98)^{* *}\end{array}$ & $P=0.038$ & - & - & - \\
\hline
\end{tabular}

Atezolizumab was initially evaluated in a phase II randomized trial [POPLAR study], where 277 patients were randomized to receive atezolizumab $1,200 \mathrm{mg}$ or docetaxel every 3 weeks [19]. It included 97 patients with Sq NSCLC of which 49 received
Atezolizumab. Atezolizumab improved over all survival in patients with Sq NSCLC by 1.5 months (HR 0.80; 95\% CI 0.49-1.30). This was inferior to improvement seen in the non-squamous NSCLC (HR 0.69; 95\% CI 0.47-1.01) [19]. OAK study evaluated atezolizumab 
$200 \mathrm{mg}$ or docetaxel at standard dose after failure of platinumbased first line chemotherapy. PD-L1 expression was evaluated not only in TC but also in tumor infiltrating immune cells (IC) in order to recognize a combinatorial marker that could predict response to immunotherapy (Table 3). It included 222 patients with squamous and 628 patients with non-squamous histology [18].For subpopulation of Sq NSCLC [26\%], atezolizumab failed to provide any benefit. Actually docetaxel was found to be associated with better OS ( 8.9 vs. 7.7 months, HR: 0.73 ; $95 \%$ CI: 0.54 to 0.98 $\mathrm{p}=0.038$ ) [20]. However, as a group it provided improvement in OS with atezolizumab compared to docetaxel as a group. Improvement seen as a group was of 4.2 months (13.8 months vs. 9.6 months; HR 0.74, 95\% CI: 0.63-0.87; $\mathrm{p}=0.0004$ ) [20].

\section{Checkpoint Inhibitors as a Third Line Therapy}

Nivolumab has also been evaluated as third line therapy.

a. CheckMate063 enrolled 117 Sq NSCLC patients who had progressed after a platinum-doublet and one more systematic treatment. Independent radiological review revealed partial response in 17 (14.5\%) of 117 patient and stable disease in 30 [26\%] patients. Median time to response was 3.3 months (IQR 2.2-4.8), and median duration of response was not reached (95\% CI 8.31-not applicable); 13 (77\%) of 17 of responses were ongoing at the time of analysis. $30(26 \%)$ of 117 patients had stable disease (median duration 6.0 months, 95\% CI $4 \cdot 7$ 10.9). Median progression-free survival was 1.9 months, with progression-free survival of $25.9 \%$ at 6 months and $20.0 \%$ at 1 year. Median overall survival was 8.2 months and overall survival at 1 year was $40.8 \%$ [21].

b. In another study, 35 patients having Sq NSCLC, progressing after first line and second line chemotherapy received, nivolumab as a monotherapy. Of 35 patients enrolled, nine patients $(25.7 \%)$ had partial response to treatment, another $10(28.6 \%)$ patients had stable disease. The median PFS was 4.2 months. The median OS with nivolumab treatment was 16.3 months with the OS rate at 1 year was $71.4 \%$. The median time to response was 2.7 months (range 1.2-5.5) in the nine patients who responded to study treatment [22].

Checkpoint Inhibitors and Management of Squamous NSCLC- Recent Advances

\section{Consolidation Therapy}

Durvalumab is indicated in patients not progressing on chemoradiotherapy.

\section{First-Line Therapy}

Patients with PD-L1 TPS $\geq \mathbf{5 0 \%}$ : Based on current evidence they should be treated with pembrolizumab as a monotherapy.

Patients with PD-L1 TPS < 50\% with good performance: They should receive pembrolizumab + paclitaxel + platinum (carboplatin).
Patients with poor performance status and PD-L1 TPS $<$ $\mathbf{5 0} \%$ to $\geq \mathbf{1} \%$ : The may be administered pembrolizumab due to its better tolerance.

\section{Maintenance Therapy}

Pembrolizumab, if used as first line therapy should be used as maintenance therapy also.

\section{Second-Line Therapy}

With use of checkpoint inhibitors in first line therapy, their use in second line will be limited to those who have not received it as first line. Nivolumab is the only checkpoint inhibitor studied in squamous NSCLC. Pembrolizumab and atezolizumab are evaluated as a subgroup of the cohort. Atezolizumab is inferior to docetaxel in subgroup analysis and so should be avoided. Pembrolizumab needs evaluation of PD-L1 expression and benefit seems to be lower than Nivolumab (HR for OS; 0.59 vs 0.74 ). Nivolumab is also found useful in elderly patients.

\section{Conclusion}

For management of advanced Sq NSCLC, Pembrolizumab (monotherapy) is suggested as a first line therapy in place of chemotherapy in patients expressing PD-L1 $\geq 50 \%$. It needs to be used with paclitaxel and carboplatin in remaining patients with PD-L1 $<50 \%$. Nivolumab is indicated as a second line therapy if checkpoint inhibitor is not used in first line therapy. Durvalumab is indicated for localised Sq NSCLC following chemoradiotherapy as consolidation therapy.

\section{References}

1. WHO (2012) Fact Sheets by Cancer.

2. Ettinger DS, Aisner DL, Wood DE, Wallace Akerley, Jessica Bauman, et al. (2018) NCCN Guidelines Insights: Non-Small Cell Lung Cancer, Version. J Natl Compr Canc. Netw 16(7): 807-821.

3. Brahmer JR, Govindan R, Anders RA, Antonia SJ, Sagorsky S, et al. (2018) The Society for Immunotherapy of Cancer consensus statement on immunotherapy for the treatment of non-small cell lung cancer (NSCLC). J Immunother. Cancer 6(1): 75.

4. Forde PM, Chaft JE, Smith KN (2018) Neoadjuvant PD-1 Blockade in Resectable Lung Cancer. N Engl J Med 378(21): 1976-1986.

5. Reck M, Rodríguez Abreu D, Robinson AG, Hui R, Csőszi T, et al. (2016) Pembrolizumab versus Chemotherapy for PD-L1-Positive Non-SmallCell Lung Cancer. N Engl J Med 375(19): 1823-1833.

6. Gilberto Lopes, Yi-Long Wu, Iveta Kudaba, Dariusz Kowalski, Byoung Chul Cho, et al. (2018) Pembrolizumab (pembro) versus platinum-based chemotherapy (chemo) as first-line therapy for advanced/metastatic NSCLC with a PD-L1 tumor proportion score (TPS) $\geq 1 \%$ : Open-label, phase 3 KEYNOTE-042 study. Journal of Clinical Oncology 36: 18.

7. Hellmann, Ciuleanu TE, Pluzanski A, Jong Seok Lee, Gregory A Otterson, et al. (2018) Nivolumab plus Ipilimumab in Lung Cancer with a High Tumor Mutational Burden. N Engl J Med 378(22): 2093-2104.

8. Carbone DP, Reck M, Paz Ares L, Benjamin Creelan, Leora Horn, et al. (2017) First-Line Nivolumab in Stage IV or Recurrent Non-Small-Cell Lung Cancer. N Engl J Med 376(25): 2415-2426.

9. Paz Ares LG, Luft A, Tafreshi A, Mahmut Gumus, Julien Mazieres, et al. (2018) Phase 3 study of carboplatin-paclitaxel/nab-paclitaxel (Chemo) with or without pembrolizumab (Pembro) for patients (Pts) with 
metastatic squamous (Sq) non-small cell lung cancer (NSCLC). Journal of Clinical Oncology 36: 15 .

10. Jotte RM, Cappuzzo F, Vynnychenko I, Daniil Stroyakovskiy, Delvys Rodriguez Abreu, et al. (2018) Impower 131: Primary PFS and safety analysis of a randomized phase III study of atezolizumab+ carboplatin+ paclitaxel or nab-paclitaxel vs carboplatin + nab-paclitaxel as $1 \mathrm{~L}$ therapy in advanced squamous NSCLC. Journal of Clinical Oncology 36: 18.

11. Borghaei H, Hellmann, Paz Ares, Suresh S Ramalingam, Martin Reck, et al. (2018) Nivolumab (Nivo)+ platinum-doublet chemotherapy (Chemo) vs chemo as first-line (1L) treatment (Tx) for advanced non-small cell lung cancer (NSCLC) with< $1 \%$ tumor PD-L1 expression: Results from Check Mate 227. Journal of Clinical Oncology 36: 15.

12. Antonia SJ, Villegas A, Daniel D, David Vicente, Shuji Murakami, et al. (2017) Durvalumab after Chemoradiotherapy in Stage III Non-SmallCell Lung Cancer. N Engl J Med 377(20): 1919-1929.

13. Durm GA, Althouse SK, Sadiq AA, Shadia Ibrahim Jalal, Salma Jabbour, et al. (2018) Phase II trial of concurrent chemoradiation with consolidation pembrolizumab in patients with unresectable stage III non-small cell lung cancer: Hoosier Cancer Research Network LUN 14-179. Journal of Clinical Oncology 36: 15 .

14. Brahmer J, Reckamp KL, Baas P, Lucio Crinò, Wilfried EE Eberhardt, et al. (2015) Nivolumab versus Docetaxel in Advanced Squamous-Cell NonSmall-Cell Lung Cancer. N Engl J Med 373(2): 123-135.

15. Horn L, Spigel DR, Vokes EE, Holgado E, Ready N, et al. (2017) Nivolumab Versus Docetaxel in Previously Treated Patients With Advanced NonSmall-Cell Lung Cancer: Two-Year Outcomes From Two Randomized, Open-Label, Phase III Trials. J Clin Oncol 35(35): 3924-3933.
16. Squibb BM (2018) Opdivo (nivolumab) package insert. Princet, N] Bristol-Myers Squibb.

17. Grossi F, Crinò L, Logroscino A, Stefania Canova, Angelo Delmonte, et al. (2018) Use of nivolumab in elderly patients with advanced squamous non-small-cell lung cancer: results from the Italian cohort of an expanded access programme. Eur J Cancer Oxf Engl 100: 126-134.

18. Herbst RS, Baas P, Kim DW, Felip E, Pérez-Gracia JL, et al. (2016) Pembrolizumab versus docetaxel for previously treated, PD-L1-positive, advanced non-small-cell lung cancer (KEYNOTE-010): a randomised controlled trial. Lancet Lond. Engl 387(10027): 1540-1550.

19. Fehrenbacher L, Spira A, Ballinger M, Marcin Kowanetz, Johan Vansteenkiste, et al. (2016) Atezolizumab versus docetaxel for patients with previously treated non-small-cell lung cancer (POPLAR): a multicentre, open-label, phase 2 randomised controlled trial. Lancet Lond. Engl 387(10030): 1837-1846.

20. Rittmeyer A, Barlesi F, Waterkamp D, Park K, Ciardiello F, et al. (2017) Atezolizumab versus docetaxel in patients with previously treated non-small-cell lung cancer (OAK): a phase 3, open-label, multicentre randomised controlled trial. Lancet Lond. Engl 389(10066): 255-265.

21. Rizvi NA, Mazières J, Planchard D, Stinchcombe TE, Dy GK, et al. (2015) Activity and safety of nivolumab, an anti-PD-1 immune checkpoint inhibitor, for patients with advanced, refractory squamous non-smallcell lung cancer (Check Mate 063): a phase 2, single-arm trial. Lancet Oncol 16(3): 257-265.

22. Hida T, Nishio M, Nogami N, Ohe Y, Nokihara H, et al. (2017) Efficacy and safety of nivolumab in Japanese patients with advanced or recurrent squamous non-small cell lung cancer. Cancer Sci 108(5): 1000-1006.

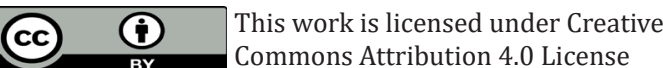

To Submit Your Article Click Here: Submit Article

DOI: $10.32474 /$ OAJOM.2018.02.000140

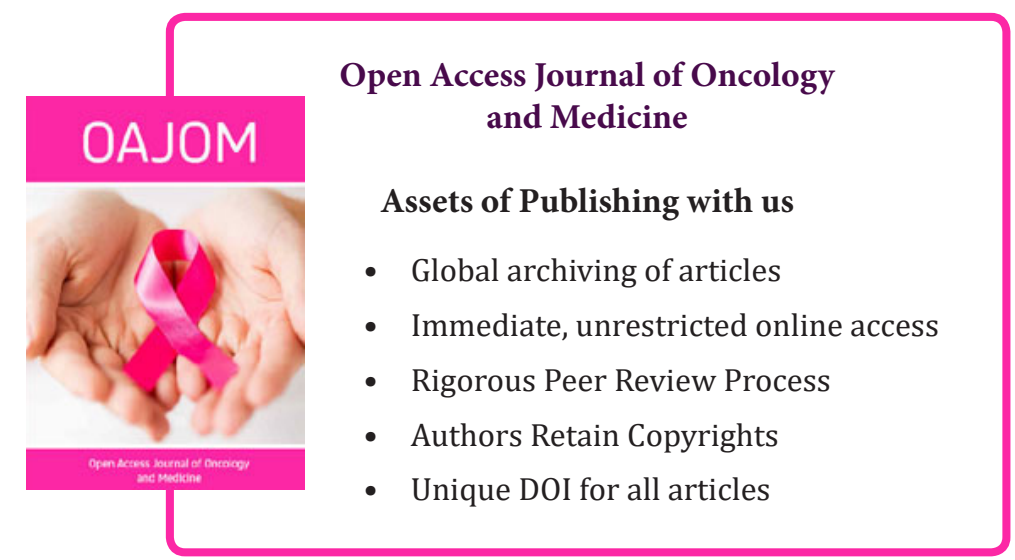

